\title{
Peritoneal Mesothelioma
}

National Cancer Institute

\section{Source}

National Cancer Institute. Peritoneal Mesothelioma. NCI Thesaurus. Code C7633.

A benign or malignant mesothelial neoplasm that arises from the peritoneum. 